\title{
What Is New in Melanoma Genetics and Treatment?
}

\author{
Simone Ribero ${ }^{a, e}$ Caterina Longo ${ }^{f}$ Dan Glass ${ }^{a, b}$ Paul Nathan ${ }^{c}$ Veronique Bataille ${ }^{a, d}$ \\ ${ }^{\mathrm{a}}$ Twin Research and Genetic Epidemiology Unit, King's College London, and ${ }^{\mathrm{b}}$ Imperial College London, London, \\ 'Melanoma Unit, Mount Vernon Cancer Centre, East and North NHS Trust Northwood, Northwood, and \\ dDermatology Department, West Herts NHS Trust, Hemel Hempstead, UK; e Section of Dermatology, Medical Sciences \\ Department, University of Turin, Turin, and fSkin Cancer Unit, Arcispedale Santa Maria Nuova, IRCCS, Reggio Emilia, Italy
}

\section{Key Words}

Genetics · Melanoma · Therapy

\begin{abstract}
New therapies for advanced melanoma have led to major advances, which, for the first time, showed improved survival for patients with this very challenging neoplasm. These new treatments are based on gene-targeted therapies or stimulation of immune responses. However, these treatments are not without challenges in terms of resistance and toxicity. Physicians should be aware of these side effects as prompt treatment may save lives. Melanoma genetics is also unravelling new genetic risk factors involving telomere genes as well as new gene pathways at the somatic level which may soon become therapeutic targets. It is also shedding new light onto the pathology of this tumour with links to neural diseases and longevity.

(C) 2016 S. Karger AG, Basel
\end{abstract}

\section{What Is New in the Genetics of Cutaneous Melanoma?}

Whole-genome sequencing of tumour DNA, expression arrays and whole-genome methylation have been bringing up a large amount of data over the last few years

\section{KARGER}

E-Mail karger@karger.com

www.karger.com/drm as seen with other cancers. It is clear that melanoma pathology involves many gene pathways, and trying to identify driver mutations is not an easy task.

\section{Susceptibility Genes}

\section{High-Risk Genes}

Melanoma high-risk genes are defined as genes that when mutated in an individual confer a high risk of developing melanoma and are usually associated with multiple melanoma cases within the family. Cyclin-dependent kinase inhibitor 2A (CDKN2A) was the first gene associated with melanoma susceptibility.

CDKN2A (p16) has been found to be relevant for around $25 \%$ of melanoma families but the prevalence of these mutations in the sporadic melanoma is very low so genetic testing is reserved for families at high risk [1]. Specific inclusion criteria for genetic testing for p 16 or $C D K 4$ (another gene linked to p16) is the presence of multiple melanomas within a family, presence of pancreatic cancer in a melanoma family or presence of multiple primary melanomas even affecting one individual only. Other family cancer syndromes may be found in the context of

S.R. and C.L. contributed equally to this work.

Dr. Simone Ribero

Department of Twin Research and Genetic Epidemiology

King's College London, St. Thomas' Campus

Westminster Bridge Road, London SE1 7EH (UK)

E-Mail simone.ribero@unito.it 
melanoma susceptibility, and these families may need screening for breast, ovary or colon cancer as appropriate.

More recently, less common germline mutations in genes such as MITF, BAP1 and p14 ARF have been linked to melanoma and have explained the clustering of kidney, breast and brain tumours within some melanoma families [2]. BAP1 is located in the chromosomal region $3 \mathrm{p} 21$ and encodes a protein that acts as a tumour suppressor through transcription regulation via chromatin remodelling and the ubiquitin-proteasome system. The frequency of CDKN2A wild-type melanoma-prone families with mutations in $B A P 1$ is not well established, but beyond cutaneous melanoma, families bearing BAP1 mutations seem to suffer also more frequently from other cancers such as uveal melanoma, mesothelioma, kidney, other cutaneous tumours and other cancers [3]. It is therefore important to document a family history of all cancers and not just skin cancer when screening patients at high risk of melanoma.

Genome-wide analyses using a case-control design have reported many more common low-penetrance single-nucleotide polymorphisms associated with increased melanoma risk [4]. Many of these genes can be grouped into pigmentation or naevus genes but many others are not really linked to specific skin phenotypes. Some of these genes are involved in telomere biology and neural diseases for example. Although pigmentation may affect the ability to tan, there are other factors associated with fair skin that may also affect melanoma risk such as body weight and immune responses via neuro-endocrine factors through the very complex melanocortin pathways $[5,6]$.

A high number of naevi, the strongest phenotypic risk factor for melanoma, has been found to be associated with longer telomeres in circulating white cells [7].

Telomeres consist of tandem nucleotide repeats (TTAGGG) and are located at the ends of chromosomes, which guard against loss of genetic material during cellular replication. Due to an inherent end replication problem, chromosomes are exposed to a potential loss of genetic material, with telomeres acting as a buffer against loss of chromatin. Repeated cell cycles eventually lead to a critically shortened telomere length, which signals cellular senescence and triggers apoptosis. This arrest in proliferation is thought to protect against malignant transformation, and a failure to do so results in catastrophic genomic instability and carcinogenesis [8].

Telomere length in white cells is predictive of biological ageing and is seen as 'biological age clock'. Telomeres shorten both with age and following environmental ex- posures associated with cancer risk, such as increased calorie intake, smoking, low bone mineral density and ultraviolet irradiation [9-11].

Several publications have now replicated the association with longer telomeres in melanoma cohorts: both telomere length and single-nucleotide polymorphisms predicting telomere length are important for predicting naevus number and melanoma risk. Horn et al. [12] identified a germline mutation in the promoter of the telomerase reverse transcriptase (TERT) gene in a melanoma-prone family with many melanoma cases using multipoint linkage analyses and target-enriched highthroughput sequencing. TERT is located in 5p15 and encodes the catalytic subunit of the telomerase, which is the ribonucleoprotein complex that maintains telomere length. However, other genes also maintain telomere length [13]. Another gene which controls telomerase is POT1, which plays an important role in telomere maintenance by preventing the inappropriate processing of the exposed chromosome ends [14]. POT1 is located within the $7 \mathrm{q} 31$ chromosomal region and encodes a protein of the telomeric shelterin complex. Two other telomere genes have been described in melanoma-prone families involved in the shelterin complex, ACD and TERF2IP [15]. Overall, germline mutations in genes involved in telomere maintenance (TERT, POT1, ACD and TERF2IP) may explain around $1 \%$ of familial melanoma cases, showing the relevance of telomere maintenance in melanoma susceptibility [16]. However, methylation of telomere genes and other mechanisms affecting telomere maintenance other than mutations need to be investigated.

This suggests that individuals at risk of melanoma with a large number of naevi may have reduced senescence and potentially increased longevity of melanocytes. So in clinical practice, this may translate as having a large number of naevi in patients aged over 50 years, and this should be regarded as a significant risk factor for melanoma.

\section{Low- to Moderate-Risk Genes}

Melanocortin 1 receptor $(M C 1 R)$ is considered a moderate-risk gene, and its role in melanoma susceptibility has been widely studied [17]. MC1R, located on 16q24, is one of the master regulator genes of human pigmentation and encodes the $\alpha$-melanocyte-stimulating hormone receptor 1 . Variants in $M C 1 R$ have different functional effects, either at the level of $\alpha$-melanocyte-stimulating hormone binding or cAMP signalling, resulting in changes in the ratio between eumelanin (brown pigment) and pheomelanin (red-yellow pigment, potentially mutagen- 
ic). $M C 1 R$ variants are associated with skin and hair pigmentation and, independently of their phenotypic effect, $M C 1 R$ variants are associated with an increased risk of developing melanoma [18].

\section{Somatic Melanoma Genetics}

At the somatic level in the tumour, the mitogen-activated protein kinase (MAPK) pathway is highly relevant in melanoma. Approximately $50 \%$ of melanoma tumours have activating mutations in the BRAF gene [19]. RAS mutations are also informative and prevalent in $20 \%$ of melanomas which are more likely to be fast-growing and nodular types of melanomas [20,21].

Mutations of $c$-KIT are preferentially observed in acral lentiginous melanoma and mucosal tumours [22]. Overall, $10-22 \%$ of patients with mucosal melanomas have tumours containing KIT mutations or amplifications [23]. Thus, sensitivity to KIT inhibition exists in a subset of melanoma tumours but these are not common [24].

In the clinical setting, $B R A F$ mutations are routinely screened in melanoma tumours in stage III and above but when $B R A F$ mutations are not detected, the tumour may need to be screened for NRAS, KIT and GNAQ mutations if appropriate [25].

Strategies to target melanomas that are BRAF/NRAS/ KIT wild type (triple wild type) have proven even more elusive. Mutations in the PTEN gene have been detected in a variety of human cancers including breast, thyroid, glioblastomas, endometrial, prostate and melanoma [26]. Initial studies demonstrated a mutation rate in PTEN of $30-40 \%$ in melanoma cell lines and $10 \%$ in primary melanomas [27]. PTEN is also involved in mediating growth arrest and other cellular functions of the MAPK pathway [28]. Germline mutations in this gene also predispose carriers to develop Cowden's disease, a heritable cancer risk syndrome which is extremely rare [29].

The identification of PI3K/AKT signalling as a core pathway for melanoma development and therapeutic escape suggested the possibility of cotargeting MAPK and $\mathrm{PI} 3 \mathrm{~K} / \mathrm{AKT}$ signalling in $B R A F$-mutant melanoma. Many melanoma mutations are in fact potentially actionable with drug targets, and drug development in these gene pathways is moving fast [30].

Genome-wide sequencing of melanoma tumours is generating interesting data. Melanoma is one of the most mutated cancers in terms of somatic changes as well as lung and bowel cancer, and the presence of many somatic changes in these tumours may be because of the increased interactions with the environment in the respective organs [18].

Genetics and Treatment of Melanoma

\section{What Is New in the Treatment of Cutaneous Melanoma?}

\section{Sentinel Node Biopsies and Lymphadenectomies}

Melanoma patients with intermediate and thick tumours are offered a sentinel node biopsy to identify lymph node spread as this procedure has prognostic value stratifying patients in different risk categories [31-33]. Other parameters such as mitoses and ulceration are also helpful in thinner melanomas [34]. After a positive sentinel lymph node has been detected, the current guidelines recommend a complete lymph node dissection of all involved metastatic basins but in cases with very small deposits in the lymph node, the patient may be given the choice of avoiding a lymphadenectomy [35]. The number of positive lymph nodes and its ratio represent the two most important prognostic factors in stage III melanoma patients $[36,37]$. We are still awaiting the results of the Multicenter Selective Lymphadenectomy Trial II (MSLT-II) in which patients with a positive sentinel node are randomized to completion lymphadenectomy or observation with ultrasound. Despite the risk of a false-negative sentinel lymph node [38], its role in predicting survival is still high enough to justify the procedure. Patients need to be well informed about the prognostic value of this procedure but access to adjuvant treatment for stage III melanoma may also justify the use of this procedure in the future.

\section{Metastatic Melanoma}

Advances in the understanding of signalling pathways, melanoma oncogenes and immune system regulation have recently resulted in a new generation of therapeutics that have significantly improved response rates and survival.

\section{Signalling Pathway-Directed Therapies}

Approximately $50 \%$ of melanoma tumours have an activating mutation in the BRAF oncogene, which results in the constitutive activation of the MAPK signalling pathway [39]. Potent selective antagonists of mutant $B R A F$ (vemurafenib and dabrafenib) are regarded as standard of care for metastatic BRAF-mutant melanoma [40,41]. Of at least as much clinical importance, however, is the fact that approximately $90 \%$ of patients whose cancers carry the BRAF mutation have some tumour shrinkage with targeted inhibitors and that these responses occur very rapidly. This rapid response rate can provide palliative relief for patients with significant tumour-related symptoms but usually last 6-9 months when other therapies may need to be considered [42-46]. 
Activated BRAF phosphorylates MEK, the next downstream target in the MAPK pathway. Although MEK is rarely mutated in untreated melanoma, it is a conduit for signalling, and inhibition of this target results in significant benefit in comparison to chemotherapy. Trametinib and cobimetinib are selective MEK inhibitors, and both have been shown to improve median progression-free and overall survival in comparison to chemotherapy in BRAF-mutant melanoma [46-48]. Dual inhibition of both BRAF and MEK pathways in BRAF-mutant disease is now proven to be significantly more potent than singleagent inhibition.

The side effects of these gene-targeted therapies with BRAF inhibitors (vemurafenib) include a variety of different effects with the majority occurring on the skin [49, 50]. The most common adverse events recorded in the BRIM-3 registration trial included arthralgia, fatigue, nausea, rashes, photosensitivity and cutaneous squamous cell carcinoma or kerato-acanthoma [51]. The dose was modified or interrupted due to adverse events in $38 \%$ of patients treated with vemurafenib and permanently discontinued in only $7 \%$ of the patients treated.

Clinical trials of dabrafenib, another BRAF inhibitor, began in 2009 with final results published in 2012. The most common adverse effects included cutaneous squamous cell carcinoma (11\%), fatigue (8\%) and pyrexia (6\%). Thirteen (7\%) patients required a reduction in the dose. Squamous cell carcinomas and kerato-acanthoma appeared less frequently than in patients treated with dabrafenib than with vemurafenib, although a direct comparison has never been investigated $[41,52,53]$.

To reduce these cutaneous side effects, it is recommended to use soap substitutes, emollients, keratolytics containing salicylic acid or urea, topical or oral antibiotics (in case of acne type eruptions on the face/trunk) and sunscreens applied every day even on non-sunny days as photosensitivity can be severe especially for vemurafenib.

The issue with RAF kinase inhibitors is acquired resistance caused by reactivation of the MAPK pathway. This results in a median progression-free survival of 6-8 months. BRAF inhibitors may also lead to new melanoma primary development induced by a paradoxical activation of the MAPK pathway in cells without BRAF mutations. This led to the introduction of a MEK inhibitor, trametinib, combined with a BRAF inhibitor, dabrafenib, for combination therapy. A significant improvement in the response rate ( 76 vs. $54 \%, \mathrm{p}=0.03$ ) and progressionfree survival was observed in the combination arm, compared to dabrafenib monotherapy [54] with fewer adverse events for squamous cell carcinoma (7 vs. 19\%, respec- tively), although the incidence of pyrexia ( 71 vs. $26 \%$ ) was increased by the introduction of a MEK inhibitor.

These patients under targeted gene therapies are also more likely to develop multiple keratotic lesions that may progress to kerato-acanthomas and squamous cell carcinomas, and may need a referral to the local dermatologist via the 2-week wait if the lesions are growing quickly. During treatment, patients can notice changes in size, shape and colour of naevi, and as new primary melanoma can occur, it is important to have dermatological input in oncology clinics treating melanoma [55]. This also highlights the need of a dermoscopy and digital monitoring to assess changing naevi in these patients and the early diagnosis of BRAF-inhibitor-related skin cancer (i.e. squamous cell carcinoma, etc.).

\section{Immunotherapy}

Melanoma is considered one of the most immunogenic tumours, and the role of the immune system in driving prognosis is well known even in localized tumours [5658].

Improved understanding of the control mechanisms regulating T-cell activation has led to a new generation of immunotherapies for many cancers and especially melanoma. The first checkpoint inhibitor in routine clinical use is ipilimumab, a humanized monoclonal antibody against the CTLA-4 protein, which stimulates immune responses against the tumour $[59,60]$. Ipilimumab benefits only approximately $15 \%$ of patients, and unfortunately there is currently no available biomarker that identifies which patients will derive benefit. Although it does improve median overall survival, the real attraction of the agent is the possibility of a long-term durable remission up to 5 years.

Recent attention has focused on an alternative checkpoint, PD-1, and its ligand PDL-1 [61]. The blockade of the PD-1/PDL-1 interaction is able to overcome immune resistance [62-64]: nivolumab and pembrolizumab have now been shown to be superior to chemotherapy and to ipilimumab alone in patients with advanced melanoma. They are licensed for use in the USA, and, at the time of writing, European licenses were obtained for pembroluzimab [65-67]. Thirty to forty percent of patients experience significant shrinkage of their tumours with these new agents, which is significantly higher than for ipilimumab.

The side effect profile of these drugs is severer than that of BRAF and MEK inhibitors with toxicity to the bowel, lung and liver with potential severe life-threatening colitis, pneumonitis and hepatitis. Although patients 
are instructed to contact their oncology team urgently in case of lung or gastrointestinal symptoms under immune therapies, general practitioner and emergency physicians may be faced with these emergencies, and swift actions contacting the oncology team with rapid use of high-dose steroids can be live saving. Pituitary failure is also a severe side effect of immunotherapy and needs to be recognized early. Other side effects that are less threatening are: fatigue, skin rashes (except extensive vasculitic rash which may signal a severe auto-immune reaction), vitiligo and neuropathy.

\section{Conclusions}

In primary care, it is important to identify patients most at risk of melanoma with a full skin examination looking for suspicious naevi as well as documenting whether the patient has a high number of naevi [68]. Documenting the family history of all types of cancers is also very important as melanoma can be found in cancerprone families, and cancer screening may be recommended. Documenting past sun exposure is unfortunate- ly not identifying patients most at risk of melanoma. It is likely that in the next 5 years, panels of melanoma susceptibility genes will be tested in high-risk patients to help devise follow-up strategies for dermatologists but also to give advice about other cancer screening, such as for bowel, breast or kidney cancer, which may be more prevalent in high-risk families. Melanoma treatment is changing at great pace with improved survival that had not been seen previously for this very challenging tumour. Many clinicians and cancer nurses may be faced with drug toxicity with these new drugs and need to be aware of the side effects for prompt treatment and/or referral. Although oncology units prescribing these drugs will have all the procedures in place to support melanoma patients, general practitioners and emergency physicians need to be vigilant for early signs of toxicity especially for the lung and gut as the prognosis is much improved if identified early and treated promptly.

\section{Disclosure Statement}

The authors have no conflict of interest to disclose.

\section{References}

1 Goldstein AM, Chan M, Harland M, et al: High risk melanoma susceptibility genes and pancreatic cancer, neural system tumors and uveal melanomas across GenoMEL. Cancer Res 2006;15:9818-9828.

2 Marzuka-Alcala A, Gabree MJ, Tsao H, et al: Melanoma susceptibility genes and risk assessment. Methods Mol Biol 2014;1102:381393.

-3 Martorano LM, Winkelmann RR, Cebulla $\mathrm{CM}$, et al: Ocular melanoma and the BAP1 hereditary cancer syndrome: implications for the dermatologist. Int J Dermatol 2014;53: 657-663.

4 Law MH, Bishop DT, Lee JE: Genome-wide meta analysis identifies five new susceptibility loci for cutaneous malignant melanoma. Nat Genet 2015;47:987-995.

5 Bohm M, Schiller M, Luger TA: Non-pigmentary actions of alpha-melanocyte-stimulating hormone - lessons from the cutaneous melanocortin system. Cell Mol Biol 2006;30:6168.

6 Raimondi S, Sera F, Gandini S, et al: MC1R variants, melanoma and red hair color phenotype: a meta-analysis. Int J Cancer 2008;122: 2753-2760.

7 Bataille V, Kato BS, Falchi M, et al: Nevus size and number are associated with telomere length and represent potential markers of a decreased senescence in vivo. Cancer
Epidemiol Biomarkers Prev 2007;16:1499- $>16$ Potrony M, Badenas C, Aguilera P, et al: Up1502.

8 Blasco MA: Telomere length, stem cells and aging. Nat Chem Biol 2007;3:640-649.

9 O'Sullivan RJ, Karlseder J: Telomeres: protecting chromosomes against genome instability. Nat Rev Mol Cell Biol 2010;11:171-181.

10 Valdes AM, Andrew T, Gardner JP, et al: Obesity, cigarette smoking, and telomere length in women. Lancet 2005;366:662-664.

11 Ribero S, Glass D, Aviv A, et al: Height and bone mineral density are associated with naevus count supporting the importance of growth in melanoma susceptibility. PLoS One 2015;10:e 0116863.

12 Horn S, Figl A, Rachakonda PS, et al: TERT promoter mutations in familial and sporadic melanoma. Science 2013;339:959-961.

13 Codd V, Nelson CP, Albrecht E, et al: Identification of seven loci affecting mean telomere length and their association with disease. Nat Genet 2013;45:422-427.

14 Palm W, de Lange T: How shelterin protects mammalian telomeres. Annu Rev Genet 2008;42:301-334.

15 Aoude LG, Pritchard AL, Robles-Espinoza $\mathrm{CD}$, et al: Nonsense mutations in the shelterin complex genes ACD and TERF2IP in familial melanoma. J Natl Cancer Inst 2015;107: dju408. date in genetic susceptibility in melanoma. Ann Transl Med 2015;3:210.

17 Ward KA, Lazovich D, Hordinsky MK Germline melanoma susceptibility and prognostic genes: a review of the literature. J Am Acad Dermatol 2012;67:1055-1067.

18 Williams PF, Olsen CM, Hayward NK, et al: Melanocortin 1 receptor and risk of cutaneous melanoma: a meta- analysis and estimates of population burden. Int J Cancer 2011;129: 1730-1740.

19 Davies H, Bignell GR, Cox C, et al: Mutations of the BRAF gene in human cancer. Nature 2002;417:949-954.

20 Mann GJ, Pupo GM, Campain AE, et al: BRAF mutation, NRAS mutation, and the absence of an immune-related expressed gene profile predict poor outcome in patients with stage III melanoma. J Invest Dermatol 2013; 133:509-517.

21 Goodall J, Wellbrock C, Dexter TJ, et al: The Brn-2 transcription factor links activated BRAF to melanoma proliferation. Mol Cell Biol 2004;24:2923-2931.

22 Curtin JA, Busam K, Pinkel D, et al: Somatic activation of KIT in distinct subtypes of melanoma. J Clin Oncol 2006;24:4340-4346.

23 Carvajal RD, Antonescu CR, Wolchok JD, et al: KIT as a therapeutic target in metastatic melanoma. JAMA 2011;305:2327-2334. 
24 Kirkwood JM, Bastholt L, Robert C: Phase II, open-label, randomized trial of the MEK1/2 inhibitor selumetinib as monotherapy versus temozolomide in patients with advanced melanoma. Clin Cancer Res 2012;18:555567.

25 Ponti G, Pellacani G, Tomasi A, et al: Molecular targeted approaches for advanced BRAF V600, N-RAS, c-KIT, and GNAQ melanomas. Dis Markers 2014;2014:671283.

26 Celebi JT, Shendrik I, Silvers DN, et al: Identification of PTEN mutations in metastatic melanoma specimens. J Med Genet 2000;37: 653-657.

27 Tsao H, Zhang X, Benoit E, et al: Identification of PTEN/MMAC1 alterations in uncultured melanomas and melanoma cell lines. Oncogene 1998;16:3397-3402.

28 Steelman LS, Pohnert SC, Shelton JG, et al: JAK/STAT, Raf/MEK/ERK, PI3K/Akt and BCR-ABL in cell cycle progression and leukemogenesis. Leukemia 2004;8:189-218.

29 Pilarski R, Burt R, Kohlman W, et al: Cowden syndrome and the PTEN hamartoma tumor syndrome: systematic review and revised diagnostic criteria. J Natl Cancer Inst 2013;105: 1607-1616.

30 Hambright HG, Meng P, Kumar AP, et al: Inhibition of $\mathrm{PI} 3 \mathrm{~K} / \mathrm{AKT} / \mathrm{mTOR}$ axis disrupts oxidative stress-mediated survival of melanoma cells. Oncotarget 2015;6:7195-7208.

- 31 Morton DL, Thompson JF, Cochran AJ, et al: Final trial report of sentinel-node biopsy versus nodal observation in melanoma. $\mathrm{N}$ Engl J Med 2014;370:599-609.

- 32 Quaglino P, Ribero S, Osella-Abate S, et al: Clinico-pathologic features of primary melanoma and sentinel lymph node predictive for non-sentinel lymph node involvement and overall survival in melanoma patients: a single centre observational cohort study. Surg Oncol 2011;20:259-264.

33 Ribero S, Osella-Abate S, Sanlorenzo M, et al: Sentinel lymph node biopsy in thick-melanoma patients $(\mathrm{n}=350)$ : what is its prognostic role? Ann Surg Oncol 2015;22:1967-1973.

- 34 Balch CM, Gershenwald JE, Soong SJ, et al: Final version of 2009 AJCC melanoma staging and classification. J Clin Oncol 2009;27: 6199-6206.

- 35 Van der Ploeg AP, van Akkooi AC, Haydu LE, et al: The prognostic significance of sentinel node tumour burden in melanoma patients: an international, multicenter study of 1,539 sentinel node-positive melanoma patients. Eur J Cancer 2014;50:111-120.

-36 Pasquali S, Mocellin S, Mozzillo N, et al: Nonsentinel lymph node status in patients with cutaneous melanoma: results from a multiinstitution prognostic study. J Clin Oncol 2014;32:935-941.

37 Sandro P, Andrea M, Nicola M, et al: Lymphnode ratio in patients with cutaneous melanoma: a multi-institution prognostic study. Ann Surg Oncol 2015;22:2127-2134.

- 38 Savoia P, Fava P, Caliendo V, et al: Disease progression in melanoma patients with nega- tive sentinel lymph node: do false-negative specimens entirely account for this phenomenon? J Eur Acad Dermatol Venereol 2012; 26:242-248

39 Davies H, Bignell GR, Cox C, et al: Mutations of the BRAF gene in human cancer. Nature 2002;417:949-954.

40 Chapman PB, Hauschild A, Robert C, et al: Improved survival with vemurafenib in melanoma with BRAF V600E mutation. N Engl Med 2011;364:2507-2516.

41 Long GV, Trefzer U, Davies MA, et al: Dabrafenib in patients with Val600Glu or Val600Lys BRAF-mutant melanoma metastatic to the brain (BREAK-MB): a multicentre, open-label, phase 2 trial. Lancet Oncol 2012; 13:1087-1095.

42 Das Thakur M, Salangsang F, Landman AS, et al: Modelling vemurafenib resistance in melanoma reveals a strategy to forestall drug resistance. Nature 2013;494:251-255.

43 Trunzer K, Pavlick AC, Schuchter L, et al: Pharmacodynamic effects and mechanisms of resistance to vemurafenib in patients with metastatic melanoma. J Clin Oncol 2013;31: 1767-1774.

44 Long GV, Stroyakovskiy D, Gogas H, et al: Combined BRAF and MEK inhibition versus BRAF inhibition alone in melanoma. N Engl J Med 2014;371:1877-1888.

-45 Long GV, Stroyakovskiy D, Gogas H, et al: Dabrafenib and trametinib versus dabrafenib and placebo for Val600 BRAF-mutant melanoma: a multicentre, double-blind, phase 3 randomised controlled trial. Lancet 2015;386: 444-451.

46 Larkin J, Ascierto PA, Dréno B, et al: Combined vemurafenib and cobimetinib in BRAFmutated melanoma. N Engl J Med 2014;371 1867-1876.

47 Flaherty KT, Robert C, Hersey P, et al: Improved survival with MEK inhibition in BRAF-mutated melanoma. N Engl J Med 2012;367:107-114.

48 Long GV, Stroyakovskiy D, Gogas H, et al: Combined BRAF and MEK inhibition versus BRAF inhibition alone in melanoma. $\mathrm{N}$ Engl J Med 2014;371:1877-1888.

-49 Sanlorenzo M, Choudhry A, Vujic I, et al: Comparative profile of cutaneous adverse events: BRAF/MEK inhibitor combination therapy versus BRAF monotherapy in melanoma. J Am Acad Dermatol 2014;71:11021109.

50 Dika E, Patrizi A, Ribero S, et al: Hair and nail adverse events during treatment with target therapies for metastatic melanoma. Eur J Dermatol, Epub ahead of print.

51 Chapman PB, Hauschild A, Robert C, et al: Improved survival with vemurafenib in melanoma with BRAF V600E mutation. N Engl J Med 2011;364:2507-2516.

52 Falchook GS, Long GV, Kurzrock R, et al: Dabrafenib in patients with melanoma, untreated brain metastases, and other solid tumors: a phase 1 dose-escalation trial. Lancet 2012;379:1893-1901.
53 Ascierto PA, Minor D, Ribas A, et al: Phase II trial (BREAK-2) of the BRAF inhibitor dabrafenib (GSK2118436) in patients with metastatic melanoma. J Clin Oncol 2013;31:32053211.

54 Flaherty KT, Infante JR, Daud A, et al: Combined BRAF and MEK inhibition in melanoma with BRAF V600 mutations. N Engl J Med 2012;367:1694-1703.

55 Argenziano G, Lallas A, Longo C, et al: Dormant melanomas or changing nevi? J Invest Dermatol 2014;134:1196-1198.

56 Ma MW, Medicherla RC, Qian M, et al: Immune response in melanoma: an in-depth analysis of the primary tumor and corresponding sentinel lymph node. Mod Pathol 2012;25:1000-1010.

57 Ribero S, Osella-Abate S, Sanlorenzo M, et al: Favourable prognostic role of regression of primary melanoma in AJCC stage I-II patients. Br J Dermatol 2013;169:1240-1245.

58 Ribero S, Gualano MR, Osella-Abate S, et al: Association of histologic regression in primary melanoma with sentinel lymph node status: a systematic review and meta-analysis. JAMA Dermatol 2015;151:1301-1307.

59 Hodi FS, O'Day SJ, McDermott DF, et al: Improved survival with ipilimumab in patients with metastatic melanoma. N Engl J Med 2010;363:711-723.

-60 Robert C, Thomas L, Bondarenko I, et al: Ipilimumab plus dacarbazine for previously untreated metastatic melanoma. N Engl J Med 2011;364:2517-2526.

61 Merelli B, Massi D, Cattaneo L, et al: Targeting the PD1/PD-L1 axis in melanoma: biological rationale, clinical challenges and opportunities. Crit Rev Oncol Hematol 2014;89:140-165.

-62 Hamid O, Robert C, Daud A, et al: Safety and tumor responses with lambrolizumab (antiPD-1) in melanoma. N Engl J Med 2013;369: 134-144.

63 Wolchok JD, Kluger H, Callahan MK, et al: Nivolumab plus ipilimumab in advanced melanoma. N Engl J Med 2013;369:122-133.

64 Topalian SL, Hodi FS, Brahmer JR, et al: Safety, activity, and immune correlates of antiPD-1 antibody in cancer. N Engl J Med 2012; 366:2443-2454.

65 Robert C, Ribas A, Wolchok JD, et al: Antiprogrammed-death-receptor-1 treatment with pembrolizumab in ipilimumab-refractory advanced melanoma: a randomised dosecomparison cohort of a phase 1 trial. Lancet 2014;384:1109-1117.

66 Robert C, Long GV, Brady B, et al: Nivolumab in previously untreated melanoma without BRAF mutation. N Engl J Med 2015;372:320330.

67 Postow MA, Chesney J, Pavlick AC, et al: Nivolumab and ipilimumab versus ipilimum$\mathrm{ab}$ in untreated melanoma. N Engl J Med 2015;372:2006-2017.

68 Ribero S, Zugna D, Osella-Abate S, et al: Prediction of high naevus count in a healthy UK population to estimate melanoma risk. Br J Dermatol 2016;174:312-318. 